\section{Childhood Bullous Pemphigoid}

A 4-year-old girl born of non-consanguinous marriage, presented with generalized vesiculobullous eruptions for a duration of 5 months. To start with, few small fluid filled lesions appeared over abdomen, then gradually involved the rest of the trunk, upper and lower extremities and also face, palms and soles. The lesions gradually increased in size and ruptured over few areas forming erosions. Patient also had burning sensation of mouth during food intake. There was no history of trauma-induced blisters. There was no history of drug intake prior to the onset of blisters and no history of photosensitivity was obtained. There was no past history or family history of similar illness or any other significant medical or surgical history.

Cutaneous examination revealed multiple tense vesicles, bullae and crusted erosions all over the body including face, palms and soles. (FIG. 1 and 2) Nikolsky's sign and bulla spread sign were negative. Older lesions healed with hypopigmentation without increasing in size at the periphery. Mucous membrane examination revealed erosions over hard palate and buccal mucosa. There was no regional lymphadenopathy. Routine laboratory tests were normal. Tzanck smear was negative.

Histopathological examination revealed subepidermal blister with neutrophils and fibrin. Dermis showed mild inflammatory infiltrate composed of polymorphs and few eosinophils. Provisional diagnosis of chronic bullous disease of childhood (CBDC) was made. Direct immunofluorescence (DIF) showed linear deposition of $\mathrm{IgG}$ and $\mathrm{C} 3$ at the dermoepidermal junction. DIF confirmed the diagnosis of bullous pemphigoid, childhood variety.

Close clinical differential diagnoses include CBDC, Epidermolysis bullosa, porphyria. String of pearls configuration of bullae which is seen in CBDC was not present in our case. There was no history of trauma induced blister as well as family history and nail dystrophy was absent. Thus, Epidermolysis bullosa was excluded. Porphyria was excluded by absence of photosensitivity, urine discoloration, systemic features or scarring. Finally DIF confirmed our diagnosis of bullous pemphigoid.

Bullous pemphigoid (BP) is the most common autoimmune subepidermal blistering disease. The disease typically presents in the elderly, with an onset after 60 years of age. Childhood variant of bullous pemphigoid is very rare.

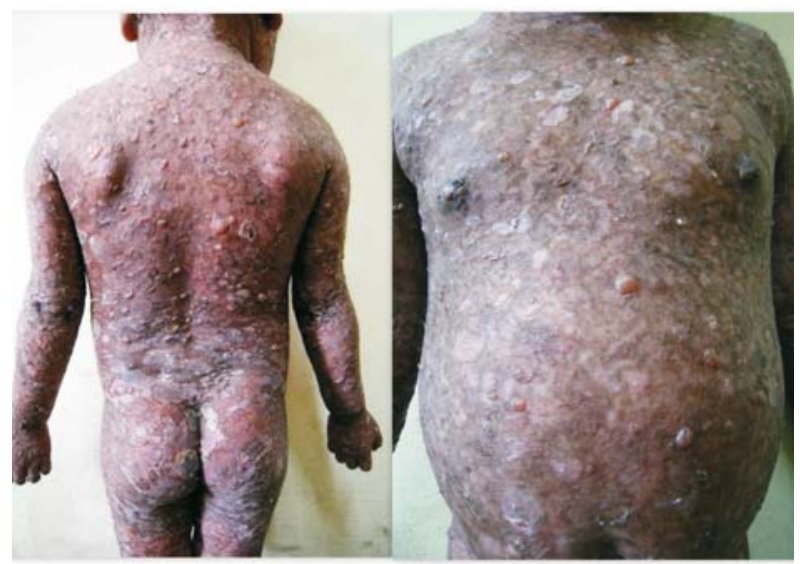

FIG. 1 Numerous blisters and erosions over the body.

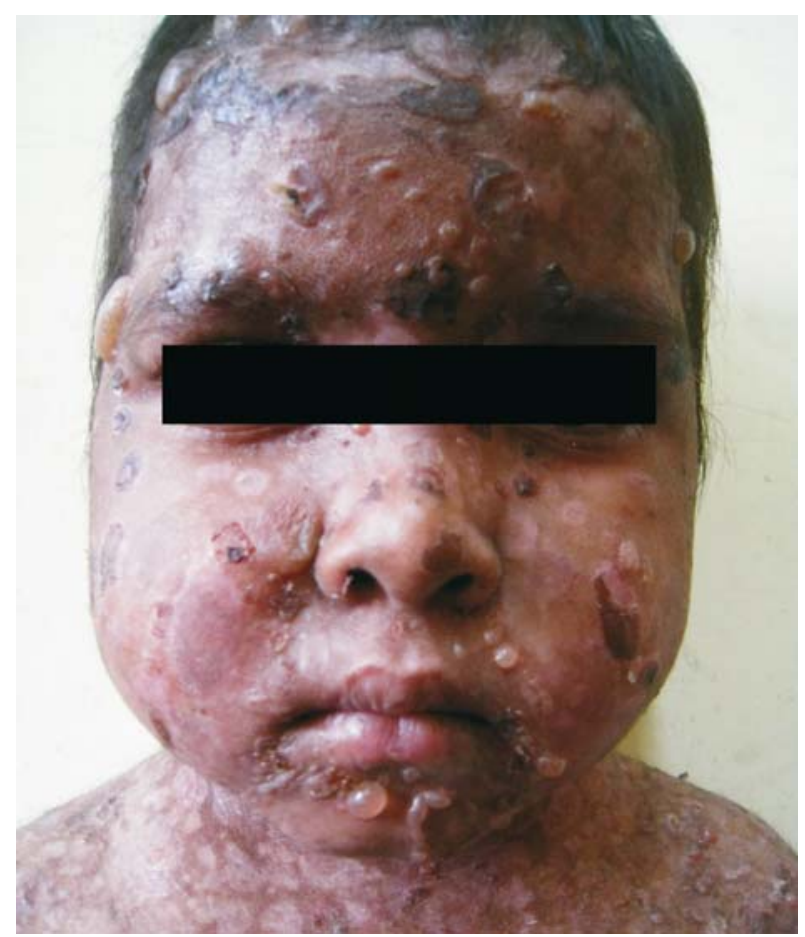

FIG. 2 Blisters and erosions over a cushingoid facies.

Systemic corticosteroids represent the first line treatment at a dose of $0.5-1 \mathrm{mg} / \mathrm{kg} /$ day. Other immunosuppressive drugs frequently used are azathioprine, methotrexate, cyclophosphamide, cyclosporine and mycophenolate mofetil. The combination of nicotinamide and tetracycline has been found to be useful. Our patient did not respond to sulphones but responded well to prednisolone which also reinforced our diagnosis.

Dipti Das, Anupam Das and Panchami Debbarman Department of Dermatology, Medical College and Hospital, Kolkata, India. anupamdasdr@gmail.com 